\title{
Museu de Ciências Morfológicas: um lugar diferente na Universidade Federal de Minas Gerais
}

\author{
The Museum of Morphological Sciences: a different place \\ at the Universidade Federal de Minas Gerais
}

Maria das Graças Ribeiro

Coordenadora Geral do Museu de Ciências Morfológicas, Universidade Federal de Minas Gerais (UFMG)

Rua Rio Grande do Norte, 300/209

30130-130 Belo Horizonte - MG mgracas@mono.icb.ufmg.br
RIBEIRO, M. G.: Museu de Ciências Morfológicas: um lugar diferente na Universidade Federal de Minas Gerais.

História, Ciências, Saúde - Manguinhos, v. 12 (suplemento), p. 339-48, 2005.

Este trabalho mostra um novo museu de ciências, cujo trabalho junto ao público é um convite enfático ao conhecimento da estrutura e funcionamento do organismo humano, como forma de entender a vida, para preservá-la com qualidade.

PALAVRAS-CHAVE: morfologia humana, educação, divulgação científica, qualidade de vida.

RIBEIRO, M. G.: The Museum of Morphological Sciences: a different place at the Universidade Federal de Minas Gerais. História, Ciências, Saúde-Manguinhos, v. 12 (supplement), p. 339-48, 2005.

The article introduces a new science museum, one that works actively to engage its audience in learning about the structure and functioning of the human organism, so that it can understand life and thus protect its quality.

KEYWORDS: human morphology, education, educational outreach in the sciences, quality of life. 


\section{O Museu de Ciências Morfológicas e sua missão}

$\mathrm{O}$ Museu de Ciências Morfológicas (MCM) foi aberto ao público em 1997, e é o único do gênero no Brasil, América Latina e Caribe. Ao contrário da maioria dos museus, cuja origem está, quase sempre, relacionada à necessidade de preservação de bens culturais e/ou coleções já existentes, o MCM resultou de um projeto de pesquisa interinstitucional desenvolvido pelo Laboratório de Histologia Animal do Departamento de Morfologia, Instituto de Ciências Biológicas da Universidade Federal de Minas Gerais (UFMG). O referido projeto constou, em sua primeira etapa, da criação e organização de coleções 'sobre o homem, para o próprio homem’. Estas coleções são apresentadas em exposições didáticocientíficas e interdisciplinares, buscando facilitar a compreensão de que a natureza a ser preservada não se encontra apenas 'lá fora', mas é parte de cada cidadão.

Entre os principais objetivos do MCM estão o de ampliar e difundir o conhecimento da estrutura e funcionamento do organismo humano, o de despertar em cada indivíduo a consciência e o compromisso de buscar novos conceitos sobre a saúde e a vida humana e ambiental, bem como o de mostrar a importância e a necessidade de preservá-las com qualidade. Tais objetivos têm sido atingidos através da linguagem museográfica de suas exposições; do trabalho cotidiano de sua equipe; do monitoramento às visitas do público escolar e da comunidade em geral; e do desenvolvimento de seus inúmeros projetos em andamento.

Motivada para responder à crescente demanda e à expectativa de públicos diferenciados, a equipe transdisciplinar do MCM vem implementando suas propostas de trabalho, dentro da visão de museu como espaço inovador, de difusão e educação científica, e não como repetidor da escola formal.

Em sua breve história, o MCM vem, na sua segunda fase, ampliando sua área física, implantando, experimentando e avaliando projetos, e apresentando expressivo crescimento quantitativo (Figura 1) e qualitativo, mensurados não só pelo aumento da demanda de público da capital e do interior (Figura 2), como também pelos resultados de seu trabalho acadêmico-institucional, didáticocientífico e social.

Além da troca de experiências com instituições museológicas do Brasil e de outros países, o MCM, por seu trabalho pioneiro nesta área, vem propiciando à comunidade um espaço de reflexão, de vivência e discussão, onde a ciência, a educação e a arte têm encontrado uma nova e harmônica forma de convivência e de estímulo. 
Figura I

PÚBLICO ATENDIDO NO MCM

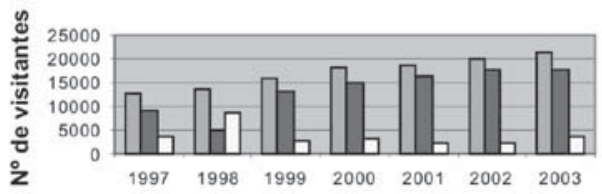

口Total de visitantes aPüblico escolar qOutros visitantes
Figura 2

INCIDENAIA DE ESCOLAS QUEVISITARAM O MCM DE 1997 A 2003

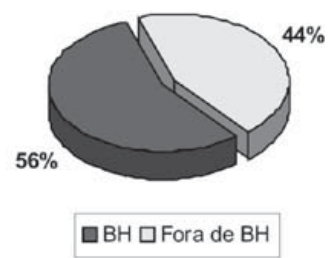

\section{As ciências morfológicas e o acervo do MCM}

Um museu de ciências morfológicas, com enfoque humano, desperta certa curiosidade, muitas vezes até pelo título. As ciências morfológicas integram áreas do conhecimento fundamentais ao entendimento da estrutura e funcionamento dos organismos, como a embriologia, que focaliza o desenvolvimento embrionário e fetal do indivíduo; a anatomia, que evidencia aspectos como forma, dimensão, constituição e localização dos diferentes órgãos e sistemas; a citologia e a histologia, que examinam a intimidade microscópica e ultramicroscópica dos organismos, possibilitando o conhecimento da organização molecular, organelas, células e tecidos, bem como o de suas interações morfofuncionais. Com acervo, portanto, bastante peculiar, o MCM mostra, através de suas exposições permanentes e itinerantes, peças anatômicas (órgãos/segmentos do corpo humano), esculturas em gesso e resinas; fotomicrografias de células e tecidos aos microscópios de luz e eletrônicos; embriões e fetos em diferentes estágios de desenvolvimento.

Equipamentos de áudio e vídeo facilitam o trabalho didático e de divulgação científica realizados pelo museu, permitindo a exibição de filmes, slides, conferências etc. As exposições são sistêmicas e a abordagem interdisciplinar, enfocando o organismo humano estrutural e funcionalmente saudável. Exposições especiais demonstram que malformações e/ou patologias podem alterar a arquitetura morfológica do homem. Algumas coleções expõem resultados de técnicas anatômicas e histológicas, confirmando a importância e a contribuição da histotecnologia, principalmente na preparação de material biológico para estudo e pesquisa. Microscópios de luz e estereoscópicos permitem a observação de células e tecidos, complementando informações sobre detalhes da organização do corpo humano. Esculturas em gesso, contribuição de conhecido artista plástico mineiro, complementam, com arte, o trabalho em prol das ciências da vida.

Rica documentação museográfica, técnica, didática e de pesquisa encontra-se à disposição de professores, pesquisadores e técnicos, visando à consulta e/ou à utilização de dados sobre o MCM (Figuras 3 a 5). 


\section{Figuras 3 a 5}

Universidade Federal de Minas Gerais
Instituto de Ciencias Biologicas - Departamento de Morfologia
MUSEU DE CIENCLAS MORFOLOGICAS (MCM)
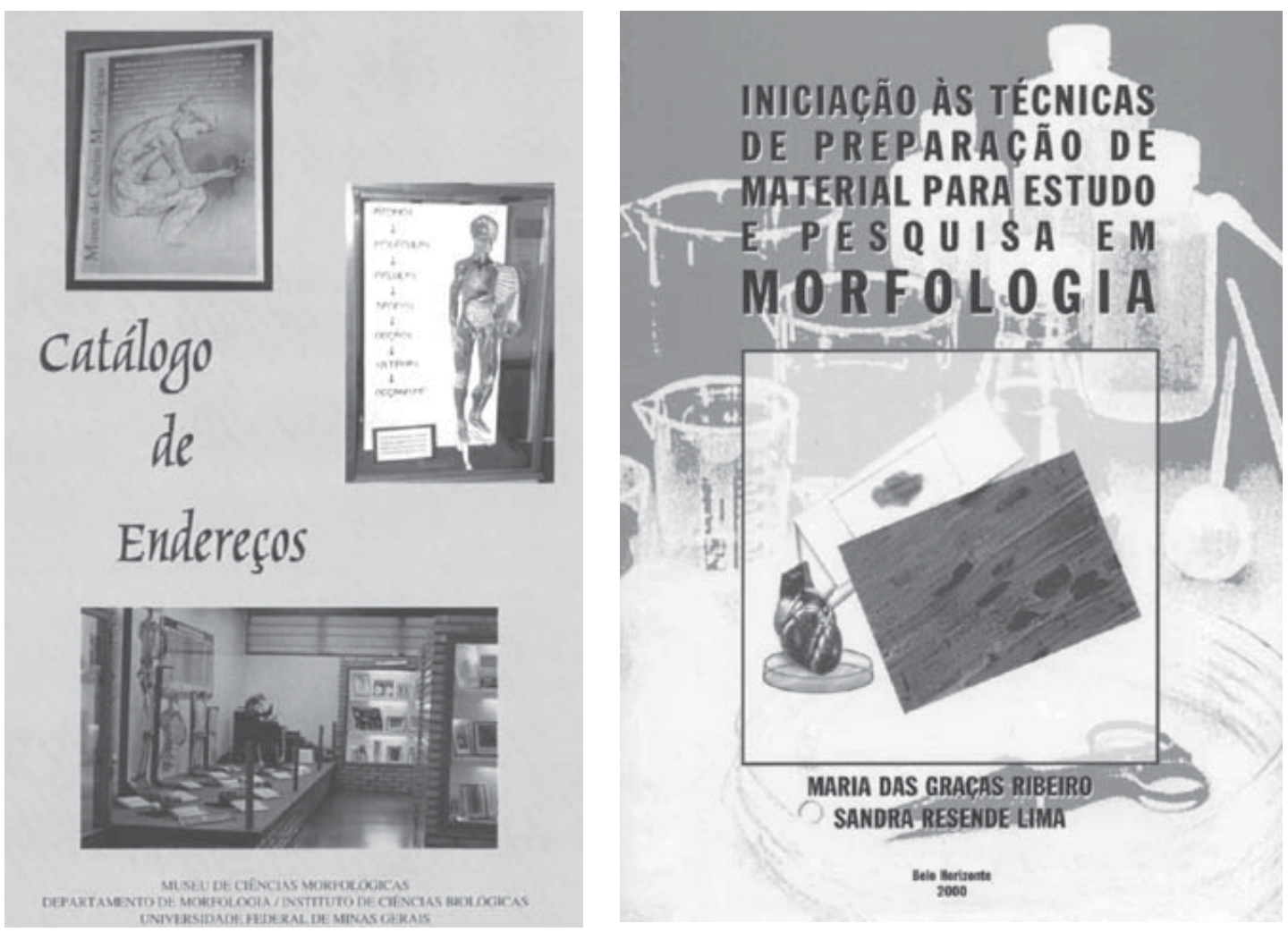


\section{Aspectos do trabalho desenvolvido no MCM}

Na implementação das atividades desenvolvidas no MCM, os limites entre ensino, pesquisa e extensão universitária foram diluídos pela integração alcançada. Em seu trabalho acadêmicoinstitucional com estudantes de graduação, o MCM reúne alunos das áreas biológica e da saúde, educação, matemática, estatística, ciência da informação, de diferentes instituições de ensino e pesquisa. Além do enriquecimento de sua formação acadêmica (ao trabalhar aspectos que vão além dos parâmetros curriculares), pessoal e interpessoal, o contato com a comunidade permite aos estagiários e monitores desenvolver a comunicação com diferentes tipos de público, preparando-se adequadamente para seu futuro desempenho profissional. Os cursos e/ou pesquisas envolvendo a pósgraduação em atividades do MCM ampliam a formação e atualização de estagiários e técnicos do museu, assim como o material gerado amplia e enriquece o seu acervo.

Em seu projeto educacional, o MCM, pela peculiaridade de seu acervo, oferece palestras, ilustradas por slides, filmes, como atividades preparatórias para as visitas monitoradas às suas exposições. Os monitores são preparados tanto para a visão interdisciplinar do conteúdo que irão abordar em diferentes níveis quanto para incentivar a curiosidade do visitante; para o esclarecimento de dúvidas e/ou orientações sobre temas específicos. Oficinas de ciências, consulta a bibliografia especializada e uso de equipamentos complementam as atividades pedagógicas do museu, oferecidas sob a orientação de diferentes especialistas da equipe do MCM ou instituições parceiras.

Em seu projeto científico, o MCM vem mostrando um novo modelo contextual de pesquisa, no qual tanto as pesquisas morfológicas quanto as pesquisas em educação, relacionadas ao ensino de ciências, partem do processo, do fazer científico, com suas dificuldades e limites, desmistificando a ciência.

No programa de divulgação científica tem sido preocupação do MCM mostrar à comunidade que divulgação científica não é apenas falar sobre o que a ciência faz, mas informar o que pode ser transformado a partir do que ela faz... Muitos dos temas divulgados no museu correspondem àqueles sugeridos e/ou levantados a partir da necessidade junto à comunidade, subsidiando a solução de problemas principalmente relacionados à saúde. Entre os temas mais solicitados estão dengue, febre amarela, doação de sangue, doação de órgãos, tabagismo, estresse, depressão, insônia, alcoolismo, uso e abuso de drogas e suas conseqüências, Aids, doenças sexualmente transmissíveis, doenças ocupacionais, dentre outros, discutidos com a comunidade principalmente através do projeto Leve Ciência para a Vida, envolvendo inúmeros pesquisadores e laboratórios da UFMG. 
Em seu projeto social, o MCM oferece palestras, conferências e cursos de educação para a saúde, alguns desenvolvidos através de exposições itinerantes. Além disso, participa de diferentes programas sociais já existentes, como Comunidade Solidária, Projeto Manuelzão, UFMG Jovem, Rede de Museus e Espaços de Ciências da UFMG, entre outros. Todas as atividades desenvolvidas no MCM visam a contribuir para incentivar o crescimento de uma nova consciência sobre saúde, cidadania e compromisso com qualidade de vida.

O programa de educação continuada em ciências foi criado para atender o maior público-alvo do museu, constituído por professores e estudantes de ensino fundamental e médio das redes públicas municipal e estadual (Figuras 6 e 7) e faculdadese/ ou escolas isoladas de ensino superior, principalmente aquelas formadoras de professores. Este programa visa a contribuir para a melhoria do ensino de ciências e de biologia, encurtando a distância entre a UFMG e estas escolas.

Pelas características expostas e por seu acervo e trabalho pouco convencionais, o MCM vem desenvolvendo seu projeto museológico, buscando, na integração com os demais museus e espaços de

Figuras 6 e 7
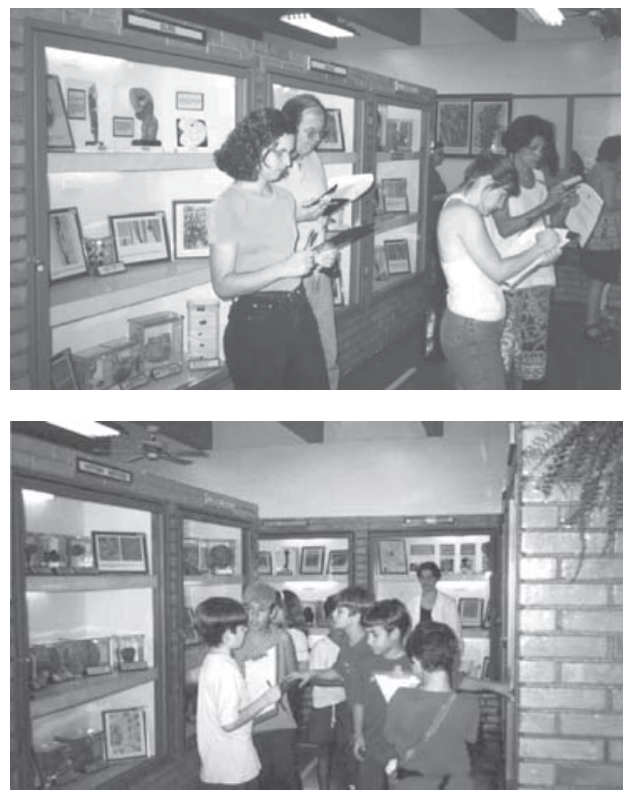
ciências, a troca de experiências e a solução de problemas que são comuns a todos. Dentre os projetos em desenvolvimento no MCM, destacam-se, principalmente, aqueles voltados para a inclusão social. Por um lado, há o esforço para, através da revitalização do ensino de ciências e do atendimento à comunidade, contribuir para a construção de uma cultura científica, na qual os resultados das pesquisas científicas e tecnológicas passem a fazer parte da vida da comunidade, subsidiando a solução de problemas da vida diária. O MCM vem implantando, nos últimos anos, projetos de inclusão social, muitos deles voltados, ainda, para a inclusão educacional de portadores de necessidades especiais de aprendizado. Criando acessibilidade às dependências do museu, portadores de deficiência física, idosos, deficientes auditivos vêm participando de suas atividades socioeducacionais. Entretanto, diante do forte apelo visual da expografia do museu, os portadores de deficiência visual só 
puderam ter acesso às suas atividades através da coleção de modelos didáticos tridimensionais do projeto "A célula ao alcance da mão" (Figuras 8 a 11).

Figuras 8 a II
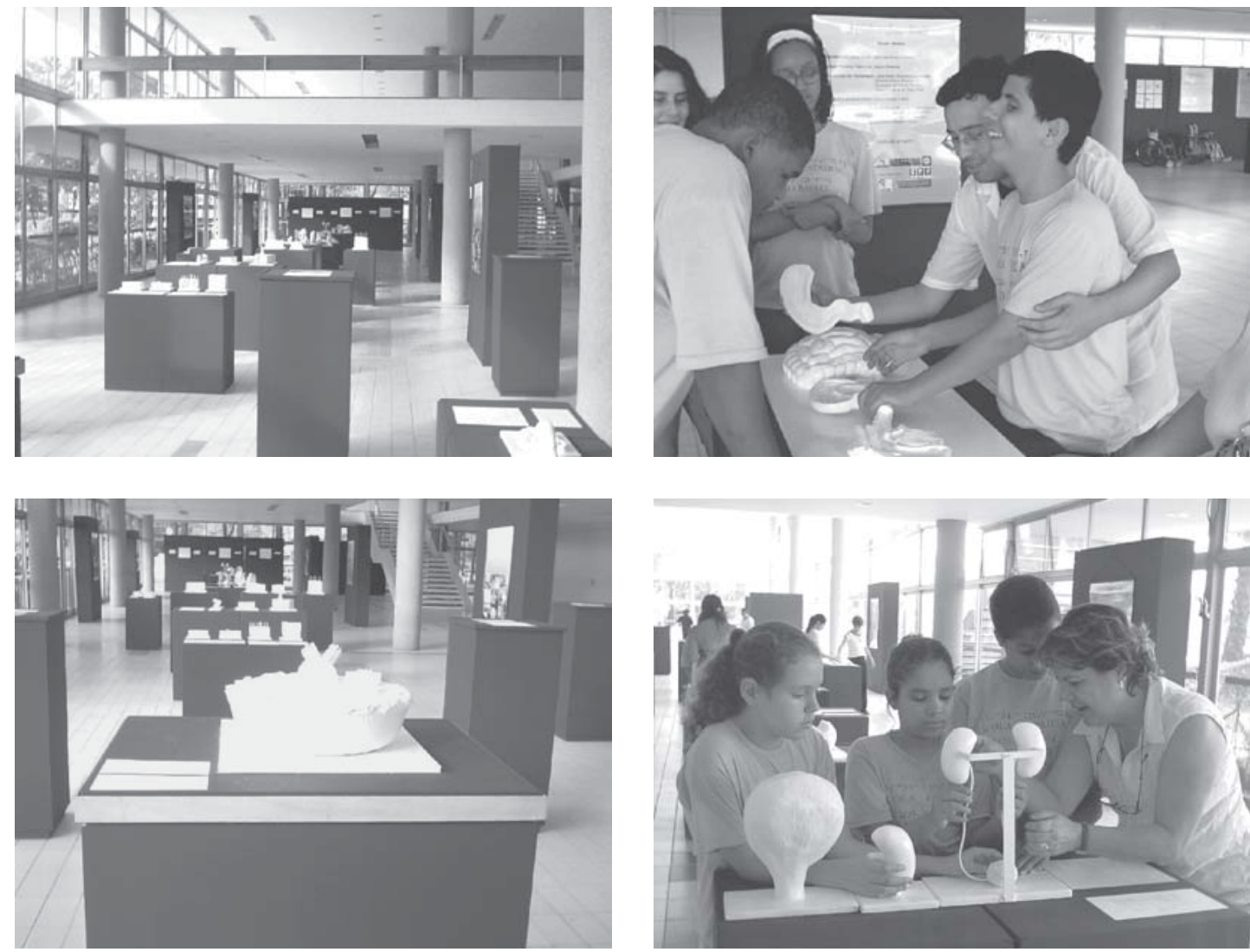

Após a fase experimental da referida coleção de modelos (propriedade intelectual da UFMG) em escolas de ensino fundamental e médio, toda a coleção didática será disponibilizada ao nosso e a outros países que dela quiserem se utilizar, visando a dar ao ensino de ciências um caráter mais dinâmico, interessante e lúdico. "Qualidade de vida não tem idade" - pesquisa e educação para o envelhecimento. "Preserve seu corpo, não o estrague" - segmento voltado para dependentes químicos, em geral menores em situação de risco e grupos empenhados em sua recuperação, dentre outros. A documentação técnica e museográfica disponibilizada para a comunidade escolar e/ou de pesquisa junto com o livro Iniciação às técnicas de preparação de material para estudo e pesquisa em morfologia (este contendo orientação inclusive sobre a ética na pesquisa) têm permitido novo tipo de intercâmbio do MCM com outras instituições, principalmente de ensino médio e cursos técnicos. E o CD-ROM, 
Agradecimentos

A autora agradece a valiosa colaboração das professoras Roseli Deolinda Ribeiro, Sandra Maria das Graças Maruch e Maria Eloiza Oliveira Teles; da bióloga Sandra Resende Lima; da bibliotecária Maria Cecília de Souza

Lima; do estagiário Jorge Nasser Júnior. além da publicação do primeiro livro virtual sobre morfologia humana, vai permitir ao museu chegar a muitas escolas, cujo acesso ao MCM não tem sido possível. A prioridade do museu, no momento, é a ampliação de seu espaço físico e de sua capacidade de atendimento.

O MCM é uma unidade museológica jovem, que tem crescido apesar dos problemas e dificuldades que enfrenta desde o início do projeto, centrados principalmente na falta de políticas próprias para os museus e centros de ciências e na falta de recursos financeiros, que acabam sempre em sobrecarga de trabalho para o grupo e insegurança quanto ao planejamento de atividades a médio e longo prazos. Mas, como disse certo pesquisador, falando do MCM: "Este é um museu de pequeno porte físico, mas com entusiasmo e sonhos de grande porte."

\section{BIBLIOGRAFIA DE APOIO}

Almeida, Adriana M. 1995

Almeida, Marcelina das Graças de 1998

Amaral, D. P. do;

Gouvêa, Guararira e

Marandino, Martha 1999

Arantes, Otília B. F. 1991

Barbosa, Cátia R. 1999

Boulilhet, Henri e

Girald, Daniéle 1990

Brefe, Ana

Cláudia Fonseca 1998

Bruno, Cristina O. 1994

Campos, Vinício Sein 1972

Cardoso, Claudia

Cristina

2000
Estudos de público: a avaliação de exposição como instrumento para compreender um processo de comunicação.

Revista do Museu de Arqueologia e Etnologia, v. 5, p. 325-34.

Museu: espaço educativo, lugar de memória.

Revista Presença Pedagógica, v. 4, n. 23, p. 69-77.

A ciência, o brincar e os espaços não formais de educação.

Rio de Janeiro, Mast/CNPq. (mimeo.)

Os novos museus. Novas construções de museus na República Federal da Alemanha. s.l., Instituto Goethe/MAC-USP. (mimeo.)

O museu de ciências: a estética e a arte: relações com o ensino de ciências.

Monografia (especialização em ensino de ciências), Belo Horizonte, Faculdade de Educação da Universidade Federal de Minas Gerais.

O museu e a vida.

Rio de Janeiro, Fundação Nacional Pró-Memória.

Os primórdios do museu: da elaboração conceitual à instituição pública.

Projeto história: trabalhos da memória. São Paulo, v. 17, p. 281-315.

Novos tempos, novos museus.

Cadernos, MAE/USP. 5 p. (mimeo.)

Elementos de museologia: história dos museus no Brasil.

São Paulo, Secretaria de Cultura, Esportes e Turismo do Governo do Estado de São Paulo.

Museu educação: subsídio para o planejamento de atividades educativo-culturais dos museus. Cadernos MHNJB - Belo Horizonte. (mimeo.) 
Carvalho, Manuel Rio 1997

Chagas, Mário 1985

Crespan, José Luis e

Trallero, Manuel 1979

Federsoni Júnior, P. A. 1998

Goldemberg, José 1998

Horta, Maria de Lourdes

Parreiras et al. 1999

Kramer, Sonia 1998

Lewis, B. N. 1980

Loomes, R. J. 1987

Lopes, Maria Margaret 1991

Lozoya, Betina e

Garcia, Andrea 1978

Meyer, Mônica A. de Azevedo 2000

Monteiro, R. Regina 1969

Nascimento, Silvânia

Souza do 2001

Nascimento, Silvânia Souza do 2001

Nascimento, Silvânia Souza do e Ventura, Paulo César Santos 2001

Neal, A. 1976

Ribeiro, Berta G. 1985

Ribeiro, Maria das Graças 1995
Problemas das visitas de ensino aos museus.

Lisboa, 6 p. (mimeo.)

Um novo (velho) conceito de museus.

Cadernos de Estudos Sociais, v. 1, n. 2, p. 183-92.

Passado e presente dos museus. In: Rojas, Roberto; Crespan, José Luis e Trallero, Manuel (orgs.). Os museus no mundo. Rio de Janeiro, Salvat (Série: Biblioteca Salvat de Grandes Temas), p. 7-59.

Museu como modelo de educação não formal.

Biológico, v. 60, n. 2, p. 79-85.

Museu de ciência. In: Centros e museus de ciências: visões e experiências.

São Paulo, Saraiva.

Guia básico de educação patrimonial.

Brasília, Instituto do Patrimônio Histórico e Artístico Nacional.

Produção cultural e educação: algumas reflexões críticas sobre educar com museu. In: Sonia Kramer e Maria Isabel F. Pereira Leite (orgs.). Infância e produção cultural. Campinas, Papirus.

The museum as an educational facility.

Museums, v. 80, p. 151-5.

Museum evaluation: new tool for management.

Nashville, American Association for State and Local History, 905p

A favor da descolarização dos museus.

Educação e Sociedade, n. 40, p. 443-55.

Tema del mes: museo y escuela.

Cuadernos de Pedagogia, n. 42, p. 10-2.

A contribuição dos museus para ensino formal de ciências. Goiânia,

Centro de Cultura e Convenções de Goiânia. (mimeo.)

Binômio museus e educação. 2 ed.

Rio de Janeiro, Museu Nacional de Belas Artes.

Novas formas de popularização da cultura científica: o exemplo da França. Presença Pedagógica, v. 7, n. 37.

O papel do organizador da ação em espaços não escolares.

Belo Horizonte, FAE/UFMG. (mimeo.)

Mutações na construção dos museus de ciências.

Campinas, Unicamp.

Exhibits for the small museum: a handbook.

Nashville, American Association for the State and Local History, 169p.

Museu: veículo comunicador e pedagógico.

Revista Brasileira de Estudos Pedagógicos, v. 66, p. 77-98.

O ensino de ciências ganha um corpo humano real no Museu de Ciências Morfológicas da UFMG. Bios, v. 2, n. 3, p. 49-52. 
Ribeiro, Maria das Graças; Teles, Maria Eloiza Oliveira e Maruch, Sandra Maria

$$
1997
$$

Ribeiro, Maria das Graças; Teles, Maria Eloiza Oliveira e Maruch, Sandra Maria

$$
1995
$$

Ribeiro, Maria das Graças; Teles, Maria Eloiza Oliveira e Maruch, Sandra Maria 1994

Santos, Maria

Cecília T. Moura 1997

Santos, Maria

Cecília T. Moura 1990

Santos, Maria

Cecília T. Moura 1987

Suano, Marlene 1986

Tavares, Regina M. Mora 1992

Trigueiros, F. dos Santos 1956

Varella, Noêmia 1989
Morphological Sciences Museum: a multidisciplinary approach to the human body improves the teaching of science. Ciência e Cultura, v. 49, n. 3 , p. 169-71.

Museu de Ciências Morfológicas (MCM): um método mais atraente de estudar o corpo humano. São Luís, Reunião Anual da Sociedade Brasileira para o Progresso da Ciência, Resumos, 47.

Museu de Ciências Morfológicas: um caminho para repensar o ensino de ciências, com enfoque na ecologia humana. In: Amae educando/ Associação Mineira de Ação Educacional. Belo Horizonte, s. n., p. 33-42.

Museu: centro de educação comunitária ou contribuição ao ensino formal? Belo Horizonte, Simpósio sobre Museologia da UFMG, 1. (mimeo.)

Repensando a ação educativa dos museus.

Salvador, Centro Editorial e Didático da Ufba.

Museu, escola e comunidade: uma integração necessária.

Brasília, Ministério da Cultura.

O que é museu?

São Paulo, Brasiliense, 101p. Coleção Primeiros Passos.

Ação cultural dos museus.

Ciências em Museus, v. 4, p. 11-3.

Museus: sua importância na educação do povo.

Rio de Janeiro, Irmãos Pongetti.

A criança e o museu.

Rio de Janeiro, Fundação Mudes.

Recebido para publicação em outubro de 2001.

Aprovado para publicação em fevereiro de 2002. 